\title{
The Association of Glomerular Filtration Rate With Echocardiographic Parameters in Chronic Kidney Disease
}

\author{
Mahmut Ozdemir ${ }^{\mathrm{a}, \mathrm{f}}$, Ramazan Asoglu ${ }^{\mathrm{b}}$, Zeki Dogan ${ }^{\mathrm{c}}$, Nesim Aladagd, \\ Tayyar Akbulut ${ }^{\mathrm{e}}$, Mustafa Yurtdas ${ }^{\mathrm{c}}$
}

\begin{abstract}
Background: Cardiovascular disease (CVD) is the primary cause of mortality and morbidity in chronic kidney disease (CKD) patients. Aortic propagation velocity (APV), epicardial fat thickness (EFT) and carotid intima-media thickness (CIMT) measurements could provide additional information on assessing renal decline in CKD patients. The study aimed to evaluate EFT, AVP and CIMT in CKD patients and then investigate the association among those parameters.

Methods: A total of $170 \mathrm{CKD}$ consecutive subjects were enrolled in the study. Patients were divided into five groups according to their estimated glomerular filtration rate (eGFR) values. Each patient underwent complete transthoracic echocardiography examination. APV, EFT and CIMT were measured for analyses. A multivariate linear regression model was used for analysis to determine the independent predictors of eGFR.
\end{abstract}

Results: The lowest APV was observed in stage IV-V, and the highest APV was observed in stage I-II $(\mathrm{P}<0.001)$. Stage IV-V patients had the highest EFT and stage I-II patients had the lowest EFT $(\mathrm{P}<$ 0.001). Moreover, the lowest CIMT was observed in stage III, and the highest CIMT was observed in stage V $(\mathrm{P}<0.001)$. eGFR was significantly and positively correlated with APV and negatively correlated with EFT and CIMT. In multivariate analyses, APV (odds ratio (OR): 0.289, P<0.001), EFT (OR: -0.135, P<0.001) and CIMT (OR:

Manuscript submitted February 3, 2021, accepted February 16, 2021

Published online February 25, 2021

${ }^{a}$ Cardiology Department, School of Medicine, Istanbul Aydin University, Istanbul, Turkey

${ }^{\mathrm{b}}$ Cardiology Department, Adiyaman University Training and Research Hospital, Adiyaman, Turkey

${ }^{c}$ Cardiology Department, School of Medicine, Istanbul Atlas University, Istanbul, Turkey

${ }^{\mathrm{d}}$ Cardiology Department, School of Medicine, Yuzuncuyil University, Van, Turkey

${ }^{e}$ Cardiology Department, Van Training and Research Hospital, Health Science University, Van, Turkey

fCorresponding Author: Mahmut Ozdemir, Cardiology Department, Bayrampasa Kolan Hospital, School of Medicine, Istanbul Aydin University, Istanbul, Turkey. Email: dragit56@hotmail.com

doi: https://doi.org/10.14740/jocmr4439
$-0.388, \mathrm{P}<0.001)$ were independent predictors of eGFR.

Conclusion: We found that APV decreased, and EFT and CIMT increased as CKD progress. The present study suggests that APV, EFT and CIMT might be incorporated with the examination of CKD patients in daily practice.

Keywords: Chronic kidney disease; Aortic propagation velocity; Epicardial fat thickness; Carotid intima-media thickness

\section{Introduction}

Patients with chronic kidney diseases (CKDs) are at higher risk for morbidity and mortality in comparison to the healthy population. Cardiovascular disease (CVD) is the primary reason for death in these patients. Early vascular aging and arterial stiffness are the most prominent changes in the arteries of CKD patients; these malformations can also be seen in the early stages of CKD [1]. Many explanations have been documented for decreased vascular compliance and increased arterial stiffness, including activation of the renin-angiotensin system, vascular calcification and endothelial dysfunction in CKD patients [2].

Arterial stiffness has been recognized as a vascular biomarker, and it describes the arterial pressure response to stroke volume changes [3]. In addition, arterial stiffness is also closely related to the propagation velocity of the pressure. Carotidto-femoral pulse wave velocity (PWV), used to identify arterial stiffening, has been documented as a strong prognosticator of severe complications in CKD patients [4]. Arterial stiffness is most prominently observed in the aorta and becomes progressively worse as CKD progresses [5]. Aortic propagation velocity (APV), measured by echocardiography, can be used to identify aortic stiffness. APV is calculated by collecting the propagation velocity from the descending thoracic aorta. Gunes et al used the APV to evaluate aortic stiffness in coronary artery disease (CAD) patients and showed that it is related to carotid and coronary atherosclerosis [6].

Epicardial adipose tissue (EAT) is found at the junction of the pericardial visceral layer and myocardium. EAT directly contacts the surface of the myocardium and coronary vessels 


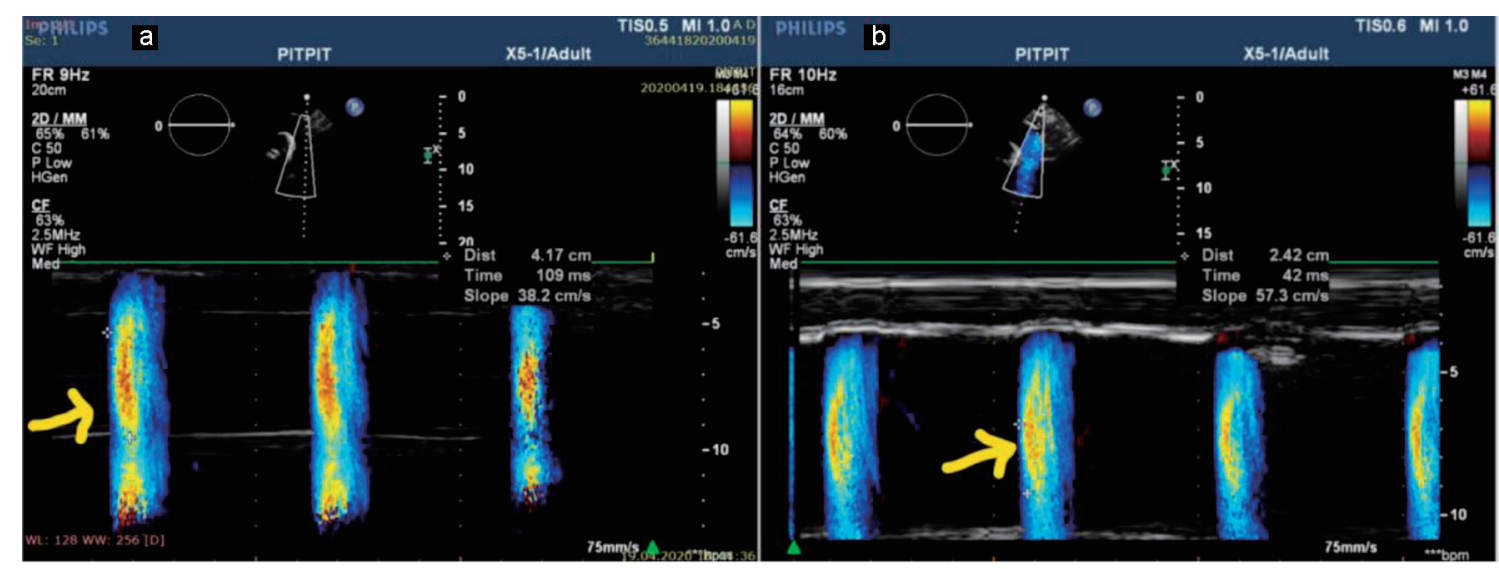

Figure 1. APV in a patient with stage V CKD $(A P V=38.2 \mathrm{~cm} / \mathrm{s})(\mathrm{a})$ and in a patient with stage III $(\mathrm{APV}=57.3 \mathrm{~cm} / \mathrm{s})(\mathrm{b}) . \mathrm{APV}$ : aortic propagation velocity; CKD: chronic kidney disease.

[7]. In addition, it secrets adipocytokines that have cardioprotective effects [8]. The pathological increase in EAT has been linked to CVD risk [9]. Many studies have proposed an association of CKD with epicardial fat thickness (EFT). Previously increased EFT has been noted in hemodialysis patients [10]. Increased EFT has been accepted as a cardiovascular risk factor in CKD patients. Nakanishi et al evaluated EAT volume using computed tomography and showed that CKD patients had more EAT compared to those without CKD [11]. Moreover, increased EFT has been linked to cardiovascular worsening in CKD [12].

Lipid accumulation in the artery causes intima-medial thickening and atherosclerotic plaque formation [13]. Carotid intima-media thickness (CIMT) has been used in the diagnosis of subclinical atherosclerosis and is associated with the presence of CAD [14]. CKD patients may be susceptible to subclinical atherosclerosis as well. The prominence in the carotid artery wall and outward remodeling has been reported in subjects with CKD [15]. CIMT has been linked to cardiovascular events and mortality in CKD patients [16]. Zuo et al showed that hypertensive CKD subjects with hypertension had augmented CIMT in comparison with non-CKD hypertensive patients [17]. Another study described that there was a negative correlation between CIMT and estimated glomerular filtration rate (eGFR) [16].

APV, EFT and CIMT measurements may indicate additional evidence in the assessment of renal decline in CKD patients. We postulate that APV, EFT and CIMT may be linked to the CKD stages. The objective of the present study was to determine the role of EFT, APV and CIMT in CKD and the relationships these parameters have in the disease.

\section{Materials and Methods}

\section{Subjects}

This study included 170 CKD subjects greater than 18 years of age. They were admitted to our clinic from September 2016 to July 2017. The criteria for CKD were based on the K/
DOQI guidelines: eGFR $\left(\mathrm{mL} / \mathrm{min} / 1.73 \mathrm{~m}^{2}\right)$ calculated by the MDRD formula [18]. Subjects were separated into five groups based on eGFR values (stage I $=76(\mathrm{eGFR}>90)$, stage II $=39$ $(\mathrm{eGFR}=60-89)$, stage III $=25(\mathrm{eGFR}=30-59)$, stage $\mathrm{IV}=$ $15($ eGFR $=15$ - 29), stage $V=15($ eGFR $<15))$. Subjects were excluded if they had uncontrolled hypertension, left ventricular (LV) systolic dysfunction, valvular pathology, effusion, acute coronary syndromes, chronic lung or hepatic disease, known malignancy, inflammatory disorders and dialysis application.

All procedures performed in this study were in accordance with the ethical standards of the institutional and/or national research committee and with the 1964 Helsinki Declaration and its later amendments or comparable ethical standards. The study was approved by the local ethics committee of the Van Training and Research Hospital.

\section{Examination}

Physical examinations were performed following an 8-h overnight fast. Blood was collected and subjected to laboratory workup. A complete transthoracic echocardiography (TTE) was recorded for each subject following the American Society of Echocardiography guidelines. The TTE (Vivid S6, GE, Norway) was performed in the resting position with the subjects facing left lateral decubitus with a $3.0-\mathrm{MHz}$ transducer. Two proficient blinded cardiologists performed the TTE. TTE images were also collected and measurements were recorded.

\section{APV analysis}

Color M-mode Doppler images were acquired while the subject was in the supine position by placing the cursor parallel to the descending aorta main directional flow. The Nyquist limit was $30-50 \mathrm{~cm} / \mathrm{s}$ and M-mode was done at a rate of $200 \mathrm{~mm} / \mathrm{s}$. If the slope of the flame was unclear, then baseline shifting was used to change the aliasing velocity until a clear delineation of the velocity slope was obtained (Fig. 1). APV was determined by dividing the distance between the beginning and end of the 


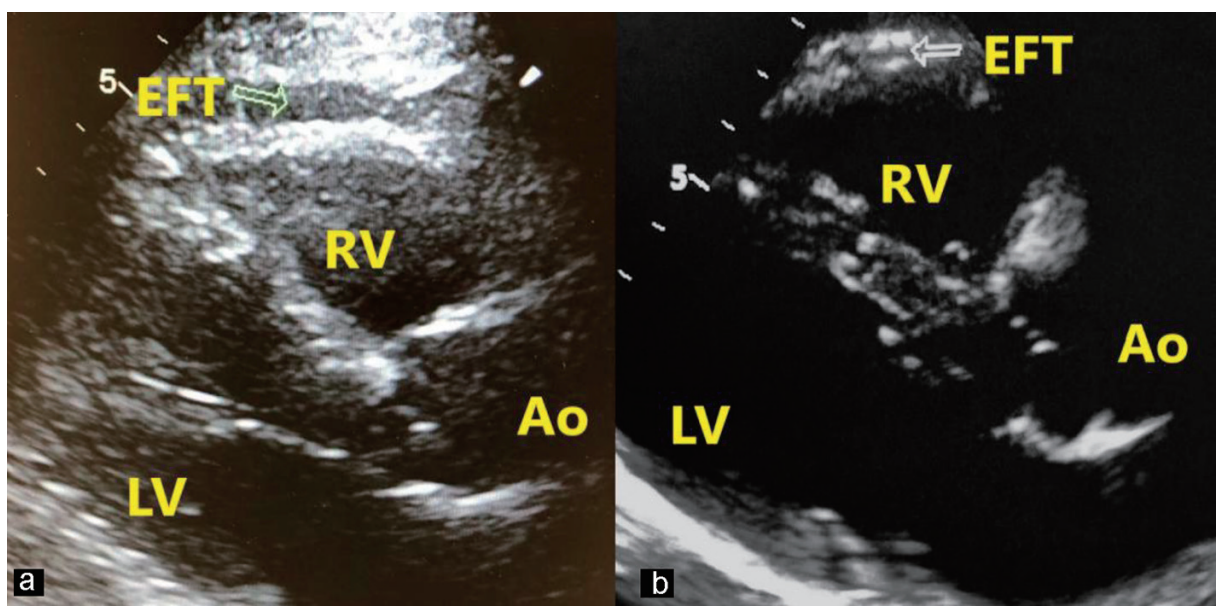

Figure 2. Measurement of EFT in a patient with stage $V(a)$ and a patient with stage $I(b)$. EFT is perpendicular to the right ventricular free wall (arrow). Ao: aort; LV: left ventricle; RV: right ventricle; EFT: epicardial fat thickness.

propagation slope by the duration. The mean of triplicate recordings was used to calculate APV [6].

\section{EFT analysis}

The EFT was measured using RV free wall from using longaxis view, while referencing the aortic annulus. The thickness of RV wall was calculated at the end-diastolic interval. The space between the visceral pericardium/myocardium was identified as the epicardial fat. To measure EFT, the thickest area was chosen to measure and the RV area was typically used (Fig. 2). The mean value of triplicate cardiac cycles was recorded [7].

\section{CIMT analysis}

Carotid arteries were assessed using a Logiq 7 (GE, Waukesha, WI, USA) with a transducer (7.5 MHz). All analyses were blinded and overseen by two knowledgeable radiologists. Measurements were recorded from the carotid arteries and included the primary transverse and longitudinal scanning of the common carotid artery, bifurcation and internal carotid. The subject was set in the supine position with their head turned to give best access for the examination. The CIMT was calculated from the frozen frame of a suitable longitudinal image $1 \mathrm{~cm}$ from the bifurcation of the common carotid artery. This was the distance from the lumen intima to the media-adventitia interface. Measurements were performed in triplicate, and the mean was recorded. All measurements made were at sites lacking plaque [14].

\section{Statistical analyses}

Data were analyzed using SPSS software v25.0 for Windows (SPSS Inc., Chicago, IL). A Kolmogorov-Smirnov test was used to test whether the data were distributed normally.
Continuous variables were expressed using mean and standard deviation. In multiple comparisons, analysis of variance (ANOVA) was used for normally distributed continuous data followed by the Tukey post hoc test. Chi-square test or Fisher exact test as appropriate with Bonferroni correction was used for categorical data comparisons. Pearson's correlation test was used for correlation analysis. Multivariate linear regression was used to analyze independent predictors. Finally, intra- and inter-observer variability was used and variables were expressed as intra-class correlation coefficients (ICCs). A Pvalue of less than 0.05 was considered statistically significant.

\section{Results}

The clinical parameters and demographics of the subjects are shown in Table 1. Subjects were assembled into five different groups based on the eGFR levels (stage I-V). Subjects in stage II-III-V were older than stage I and IV. Body mass index (BMI) and gender were not different between the groups of CKD subjects. While diabetes mellitus (DM) and smoking were similar in the groups, CAD was mostly observed in stage V. Increased hypertension and hyperlipidemia were observed in stage IV subjects. From the hematology analysis, white blood cell count and hemoglobin were the lowest in stage IV-V, while the highest creatinine value was observed in stage IV-V. Stage IV patients had the lowest $\mathrm{C}$-reactive protein value.

In the echocardiographic analysis, LV ejection fraction was similar between the CKD stages. The APV was lower in stage IV-V compared to other CKD stages (Fig. 3a). Stage IV and stage $\mathrm{V}$ subjects demonstrated increased EFT compared to the other stages $(\mathrm{P}<0.001)$ (Fig. 3b). Furthermore, increased CIMT values were observed in stage IV-V $(\mathrm{P}<0.001)$ (Fig. $3 \mathrm{c})$. The correlations among the eGFR, APV, EFT and CIMT are shown in Table 2. eGFR had a direct correlation with APV and an inverse correlation with EFT and CIMT (Figs. 4-6). APV was inversely correlated with EFT $(r=-0.401, \mathrm{P}<0.001)$ and CIMT $(\mathrm{r}=-0.628, \mathrm{P}<0.001)$. EFT values had a significantly positive association with CIMT $(\mathrm{r}=0.522, \mathrm{P}<0.001)$. 


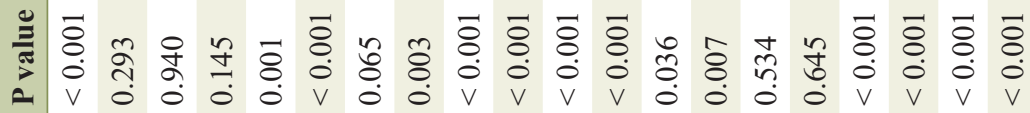

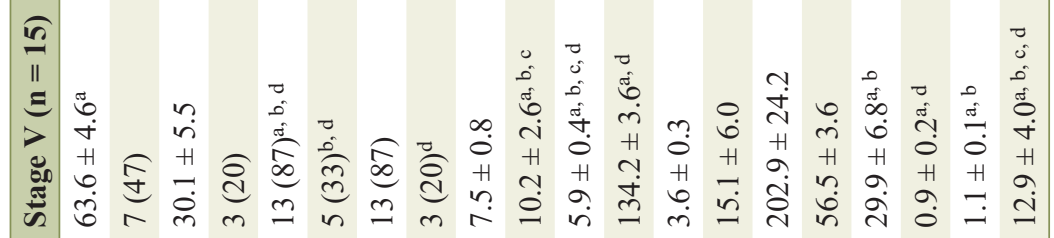

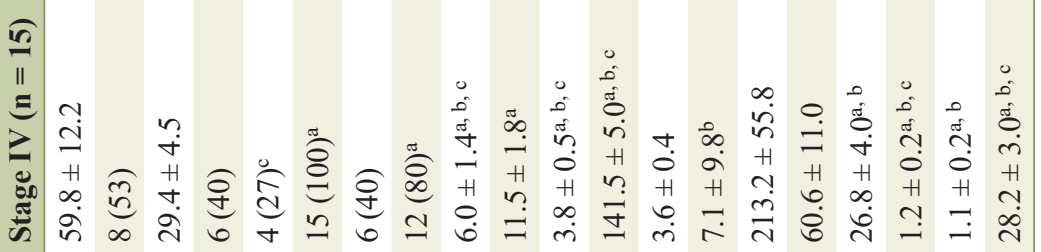

$\sqrt{3}$

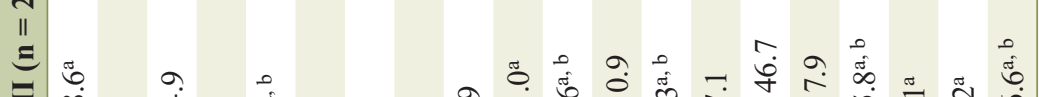

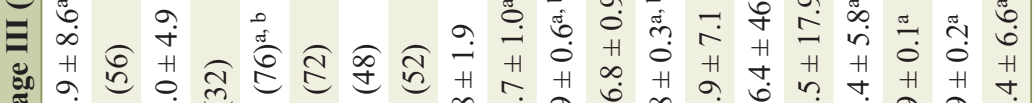

苛

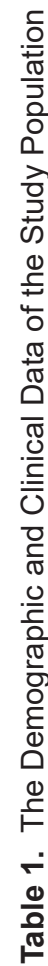

बิ

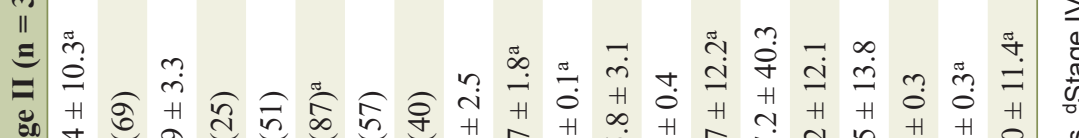

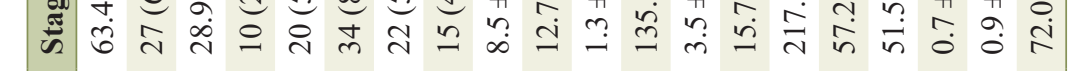

6

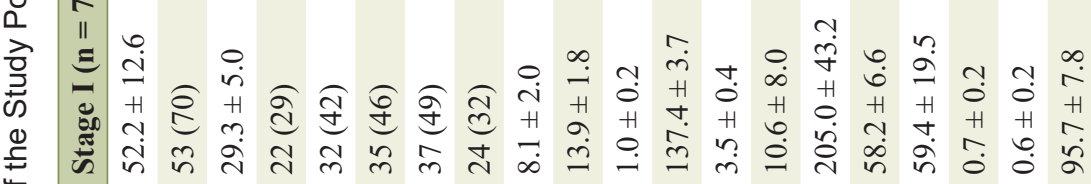
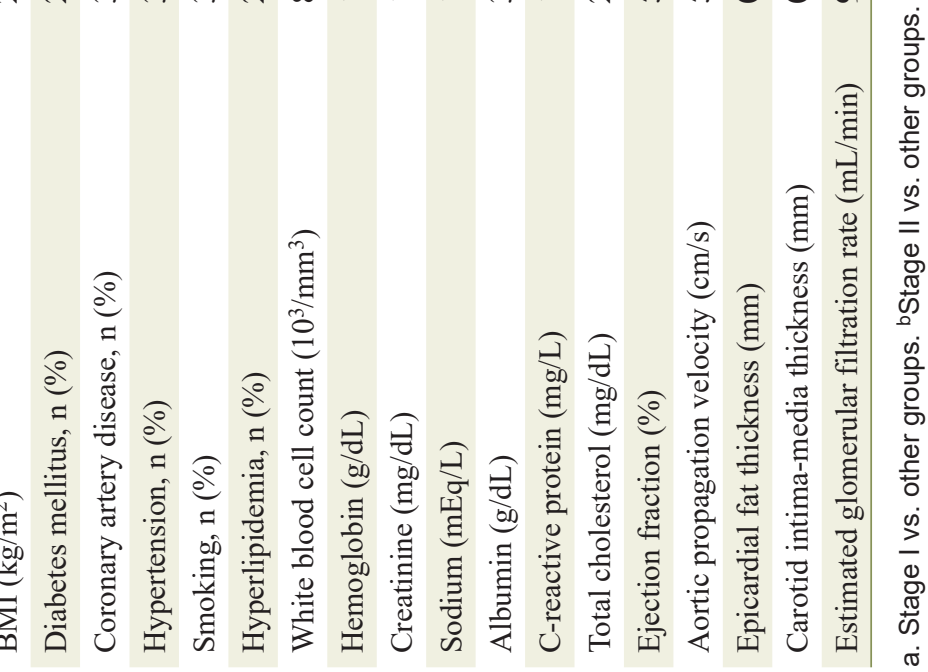


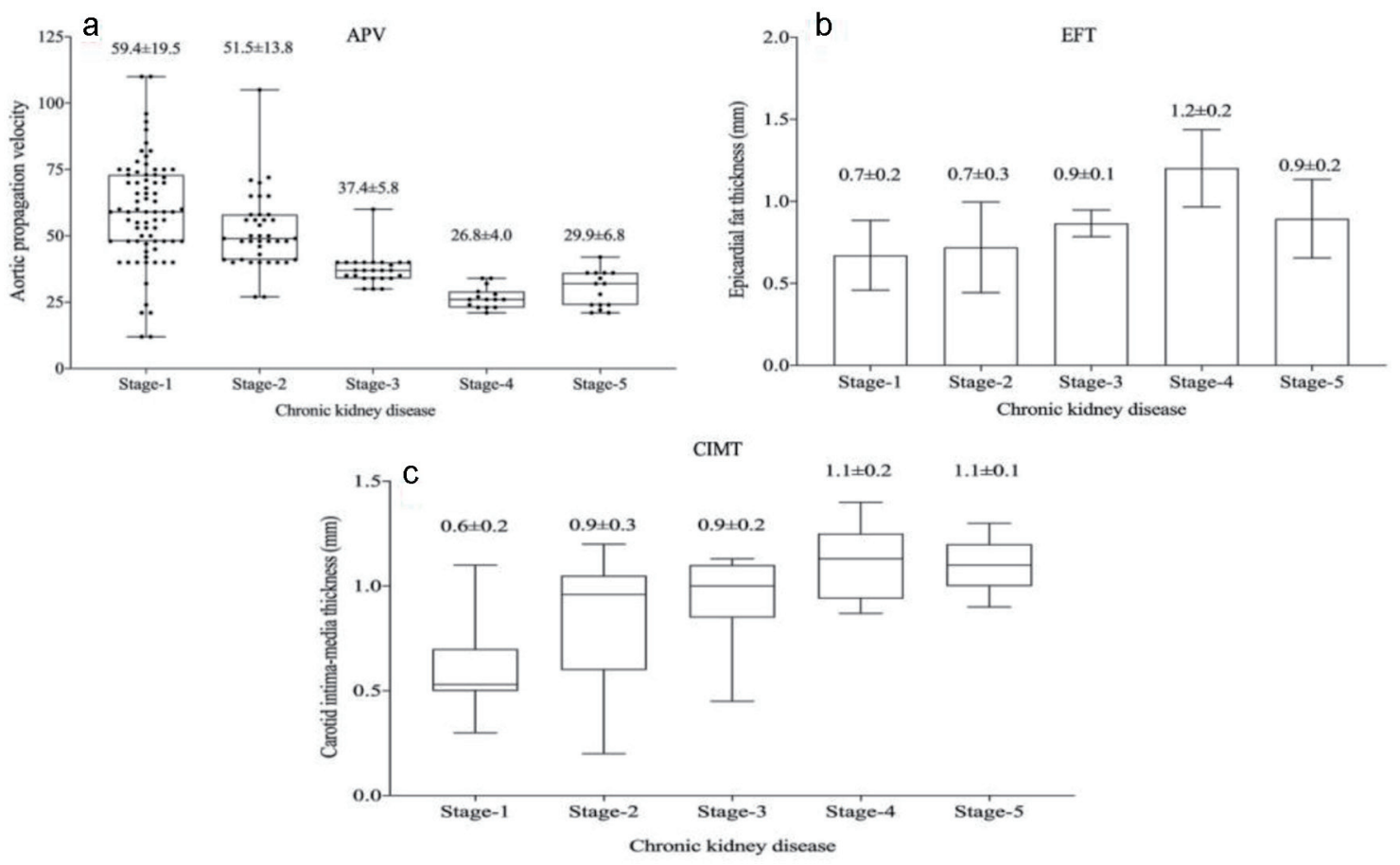

Figure 3. Aortic propagation velocity (a), epicardial fat thickness (b) and carotid intima-media thickness (c) values in chronic kidney disease stages.

Multivariate linear regression analyses of the association between the eGFR and multiple parameters are listed in Table 3. APV (odds ratio (OR): 0.291, P $<0.001$ ), EFT (OR: -0.131 , $\mathrm{P}<0.001)$ and CIMT (OR: $-0.380, \mathrm{P}<0.001)$ all predicted eGFR independently.

Intra- and inter-observer reliability of APV and EFT were evaluated in 15 subjects selected randomly and recorded as ICCs. The ICCs for APV were intra-: $0.92(95 \%$ confidence interval (CI): 0.86 - 0.95) and inter-: 0.88 (95\% CI: 0.82 - 0.94) and for EFT were intra-: 0.92 (95\% CI: 0.85 - 0.94) and inter-: 0.90 (95\% CI: 0.84 - 0.96).

\section{Discussion}

In this report, we evaluated APV, EFT and CIMT levels in the CKD stages. The key findings of this study are as follows. First, stage IV-V patients had the lower APV and higher EFT and CIMT. Second, eGFR was inversely correlated with APV and positively associated with EFT and CIMT. Third, APV was significantly and negatively associated with EFT and CIMT in CKD patients. Last but not least, APV, EFT and CIMT were independently associated with eGFR levels in CKD patients.

APV is an aortic stiffness constraint, which has been routinely recorded in electrocardiography exams. APV is correlated with aortic strain and aortic distensibility in patients with CAD [19]. Arterial stiffness is one of the early signs of cardiovascular dysfunction in CKD patients [20]. A decrease in propagation velocity can be observed with arterial stiffening in CKD patients. PWV, a marker of arterial stiffness, has been shown to independently predict CVD mortality/mortality in end-stage renal failure patients [21]. It was reported that eGFR is negatively correlated with arterial stiffness, and increased PWV could predict the presence of CKD [17]. Reduced arterial stiffness improved life expectancy in end-stage renal failure patients regardless of blood pressure status [22]. It is demonstrated that stage IV-V subjects had the lowest APV. This result is compatible with the work by Wang et al, who found that ar-

Table 2. Correlation Analysis Among eGFR, APV, EFT and CIMT

\begin{tabular}{|c|c|c|c|c|c|c|c|c|}
\hline & \multicolumn{2}{|c|}{ eGFR } & \multicolumn{2}{|c|}{ APV } & \multicolumn{2}{|c|}{ EFT } & \multicolumn{2}{|c|}{ CIMT } \\
\hline & $\mathbf{r}$ & $\mathbf{P}$ & $\mathbf{r}$ & $\mathbf{P}$ & $\mathbf{r}$ & $\mathbf{P}$ & $\mathbf{r}$ & $\mathbf{P}$ \\
\hline eGFR & - & - & 0.587 & $<0.001$ & -0.453 & $<0.001$ & -0.640 & $<0.001$ \\
\hline EFT & -0.453 & $<0.001$ & -0.401 & $<0.001$ & - & - & 0.522 & $<0.001$ \\
\hline CIMT & -0.640 & $<0.001$ & -0.628 & $<0.001$ & 0.522 & $<0.001$ & - & - \\
\hline
\end{tabular}

eGFR: estimated glomerular filtration rate; APV: aortic propagation velocity; EFT: epicardial fat thickness; CIMT: carotid intima-media thickness. 


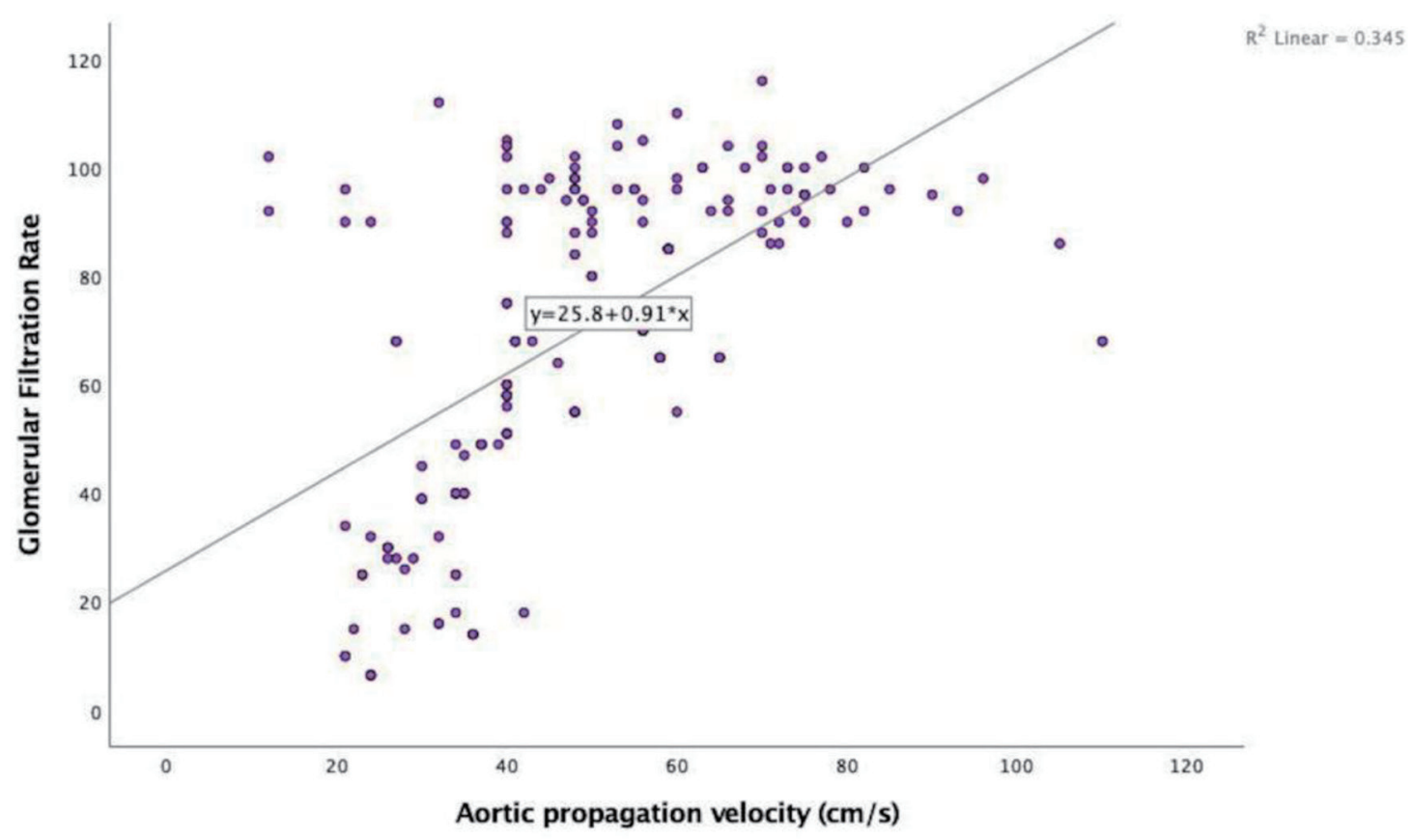

Figure 4. Correlation analysis between estimated glomerular filtration rate and aortic propagation velocity $(r=0.587, P<0.001)$.

terial stiffness gradually increased over time as CKD progress. Moreover, our finding shows that arterial stiffness increases gradually as eGFR decreases. APV was significantly and positively correlated with eGFR levels, which demonstrates the probable injury of large vessels in CKD subjects. Due to the negative correlation among the APV, EFT and CIMT levels in CKD patients, APV can be considered as a surrogate marker in subclinical atherosclerosis.

EAT contributes to cardiac function via paracrine and vasocrine secretion of pro-inflammatory/pro-atherogenic factors into the circulatory system. EAT has its protective effects in healthy people, such as mechanical protection, regulation of coronary flow and energy supplementation of fatty acids. The pathological increase in EFT has been linked to

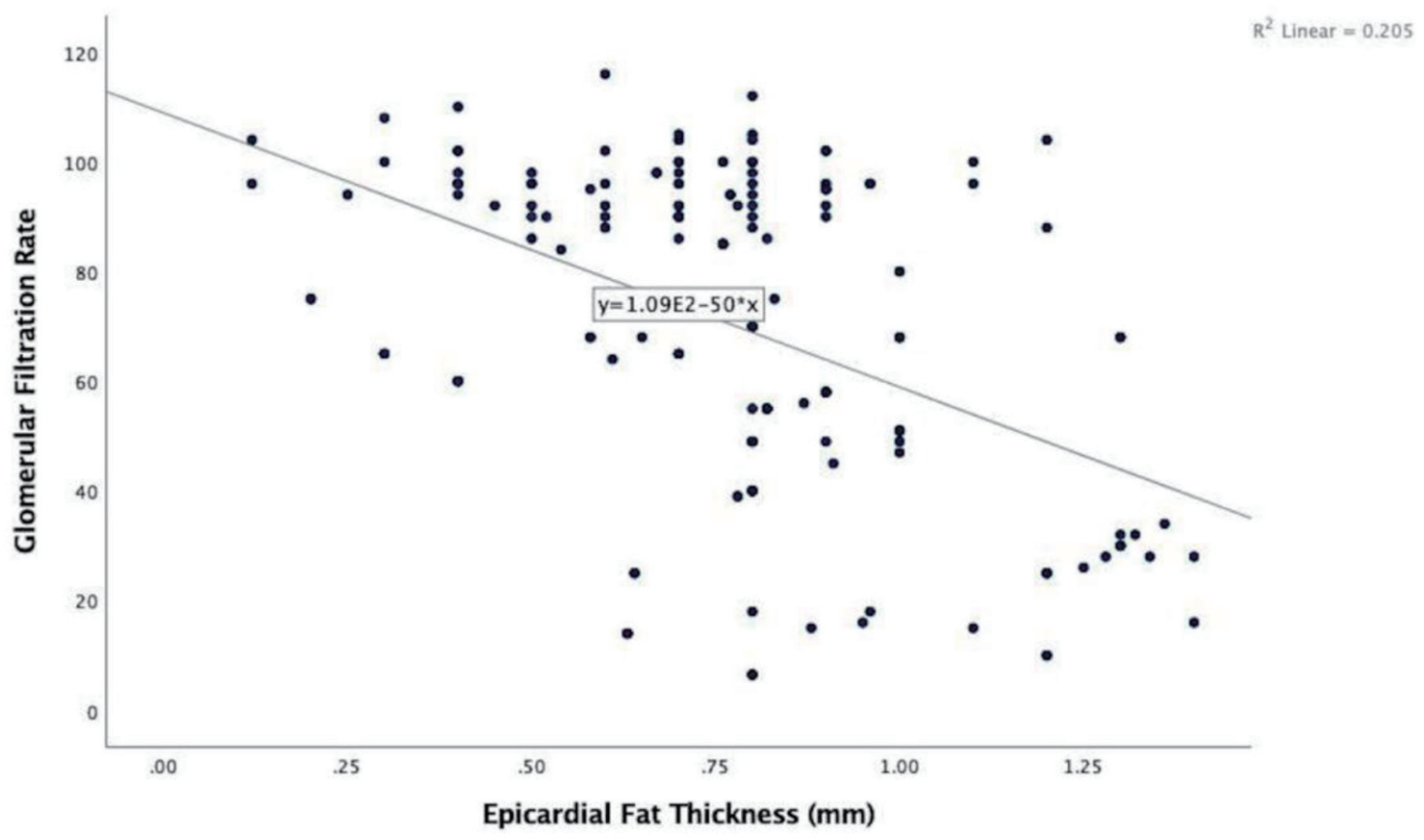

Figure 5. Correlation analysis between estimated glomerular filtration rate and epicardial fat thickness $(r=-0.453, P<0.001)$. 


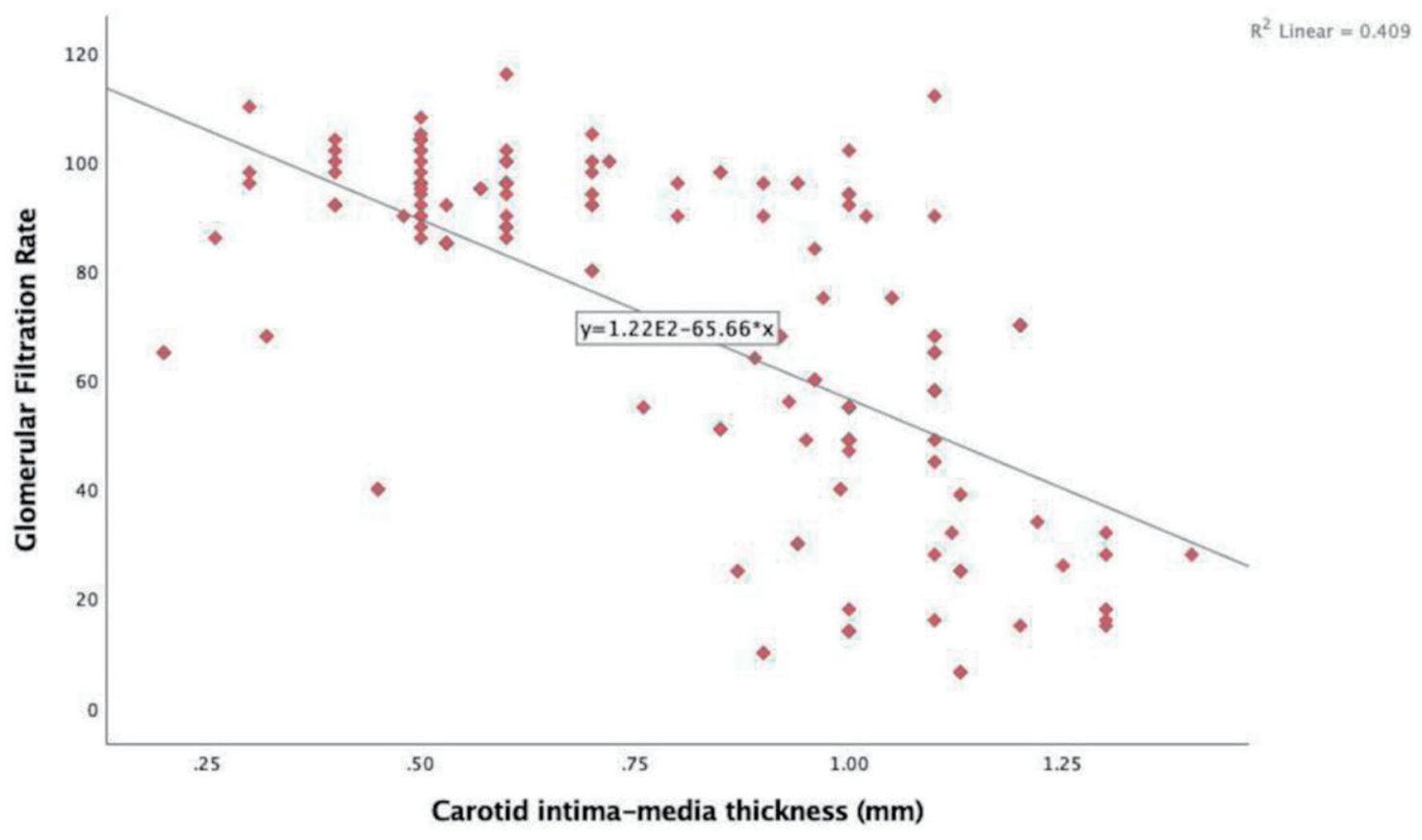

Figure 6. Correlation analysis between estimated glomerular filtration rate and carotid intima-media thickness $(r=-0.640$, $P<$ 0.001).

elevated CVD risk in CKD patients [9]. The reason for the increase in EFT is not fully understood in CKD patients. It is thought that increased inflammation may contribute to the increased EFT in these patients [23]. Aydin et al reported that hemodialysis was an independent predictor of increased EFT [24]. Patients with essential hypertension and microalbuminuria had higher EFT compared to hypertension patients with normoalbuminuria [25]. Another study proposed that EFT was an independent predictor of albuminuria in type $2 \mathrm{DM}$ patients [26]. Cordeiro et al evaluated the EFT in 227 nondialysis CKD patients and reported that EFT correlated with CVD severity. Moreover, the authors found that increased EFT was related to poor cardiovascular prognosis [12]. A meta-analysis, including the results of 17 studies, suggested that EFT and epicardial fatty volume were increased in CKD patients compared to the control group [27]. Sheng et al evaluated epicardial fatty volume by computed tomography in $120 \mathrm{CKD}$ patients and 30 healthy subjects. They found that stage IV and V patients had higher epicardial fatty vol- umes compared to the controls [28]. Despite this, Ozkurt et al showed that EFT was similar in CKD patients and controls [29]. In our study, we found that stage IV and V subjects had the higher EFT, which is consistent with a meta-analysis by Song et al [27]. In addition, we reported that EFT was inversely correlated with APV. The result is similar to work done by Turan et al [30].

CIMT has been shown to be increased in previous reports on CKD patients. The increased CIMT was associated with low-grade systemic inflammation in CKD patients [31]. Higher CIMT was associated with progressively decreasing eGFR. Moreover, in CKD patients, eGFR could predict the increase in CIMT [16]. CIMT is linked to increased mortality in CKD patients [32]. A previous study showed that CIMT was higher in hemodialysis subjects when compared with controls [24]. CIMT is independently associated with microalbuminuria in type $2 \mathrm{DM}$ patients [33]. Patel et al investigated CIMT in 62 CKD patients and 50 controls. They found that CIMT was increased in CKD subjects in comparison to controls.

Table 3. Independent Predictors for Estimated Glomerular Filtration Rate by Multivariate Linear Regression Analysis

\begin{tabular}{llll} 
& Standardized coefficients beta & t & P \\
\hline BMI & -0.032 & -0.561 & 0.576 \\
APV & 0.291 & 3.969 & $<0.001$ \\
EFT & -0.131 & -1.967 & 0.048 \\
CIMT & -0.380 & -4.694 & $<0.001$ \\
Diabetes mellitus & -0.043 & -0.757 & 0.450 \\
Hypertension & -0.019 & -0.322 & 0.748 \\
\hline
\end{tabular}

BMI: body mass index; APV: aortic propagation velocity; EFT: epicardial fat thickness; CIMT: carotid intima-media thickness. 
Furthermore, the authors showed that dialysis patients had higher CIMT compared to stage III and V [31]. Another study indicated that HT patients with CKD had significantly higher CIMT compared to HT patients without CKD [17]. In contrast, Margekar et al found no significant differences in CIMT at the different stages of CKD [34]. In the current study, we showed that CIMT is significantly and negatively correlated with eGFR. This finding is consistent with previous findings in the literature [16]. Similar to the results of this study, Patel et al observed the higher CIMT in stage V patients [31].

\section{Limitations}

This study does have a few limitations. It was single-center study examining only a small cohort and may not be sufficient to generalize the results. Patients on hemodialysis were also excluded; therefore, the validity of the results for different populations is potentially limited. In addition, EFT was not validated by other means including magnetic resonance imaing or computed tomography. Another limitation was that the association with APV and PWV was not evaluated. A large randomized clinical trial is needed to provide strength to present study findings.

\section{Conclusion}

In conclusion, APV was reduced, while EFT and CIMT were higher in CKD as the disease worsens in our study. Our findings suggest that increased EFT and CIMT, and decreased APV are closely associated with reduced eGFR in CKD patients. APV, EFT and CIMT might be used for risk assessment in various stages of CKD. Further work is needed to validate the role of APV, EFT and CIMT as diagnostic parameters in CKD patients.

\section{Acknowledgments}

None to declare.

\section{Financial Disclosure}

The authors declare that they received no funding for the present study.

\section{Conflict of Interest}

The authors declare that they have no conflict of interest.

\section{Informed Consent}

Informed consent was obtained from all individual participants included in the study.

\section{Author Contributions}

All authors contributed to the concept and design of the study, as well as to the acquisition, analysis and interpretation of data. MO, NA, RA and TA performed statistical analysis. ZD, NA, RA, MY and MO drafted the manuscript. All authors critically revised the manuscript. All authors gave final approval and agreed to be accountable for all aspects of the study, ensuring its integrity and accuracy.

\section{Data Availability}

The authors declare that data supporting the findings of this study are available within the article.

\section{References}

1. London GM, Safar ME, Pannier B. Aortic aging in ESRD: structural, hemodynamic, and mortality implications. J Am Soc Nephrol. 2016;27(6):1837-1846.

2. Moody WE, Edwards NC, Chue CD, Ferro CJ, Townend JN. Arterial disease in chronic kidney disease. Heart. 2013;99(6):365-372.

3. Vlachopoulos C, Xaplanteris P, Aboyans V, Brodmann M, Cifkova R, Cosentino F, De Carlo M, et al. The role of vascular biomarkers for primary and secondary prevention. A position paper from the European Society of Cardiology Working Group on peripheral circulation: Endorsed by the Association for Research into Arterial Structure and Physiology (ARTERY) Society. Atherosclerosis. 2015;241(2):507-532.

4. Briet M, Boutouyrie P, Laurent S, London GM. Arterial stiffness and pulse pressure in CKD and ESRD. Kidney Int. 2012;82(4):388-400.

5. Ferreira JP, Girerd N, Pannier B, Rossignol P, London GM. High Pulse-wave velocity defines a very high cardiovascular risk cohort of dialysis patients under age 60 . Am J Nephrol. 2017;45(1):72-81.

6. Gunes Y, Tuncer M, Yildirim M, Guntekin U, Gumrukcuoglu HA, Sahin M. A novel echocardiographic method for the prediction of coronary artery disease. Med Sci Monit. 2008;14(9):MT42-46.

7. Nagy E, Jermendy AL, Merkely B, Maurovich-Horvat P. Clinical importance of epicardial adipose tissue. Arch Med Sci. 2017;13(4):864-874.

8. Patel VB, Shah S, Verma S, Oudit GY. Epicardial adipose tissue as a metabolic transducer: role in heart failure and coronary artery disease. Heart Fail Rev. 2017;22(6):889902.

9. Guzzardi MA, Iozzo P. Fatty heart, cardiac damage, and inflammation. Rev Diabet Stud. 2011;8(3):403-417.

10. Karatas A, Canakci E, Bektas O, Bayrak T, Bayrak A, Altinbas A, Turkmen E. Relationship of epicardial fat tissue thickness with oxidant biomarkers in chronic kidney disease. Bratisl Lek Listy. 2018;119(9):566-571.

11. Nakanishi K, Fukuda S, Tanaka A, Otsuka K, Taguchi 
H, Yoshikawa J, Shimada K. Epicardial adipose tissue accumulation is associated with renal dysfunction and coronary plaque morphology on multidetector computed tomography. Circ J. 2016;80(1):196-201.

12. Cordeiro AC, Amparo FC, Oliveira MA, Amodeo C, Smanio P, Pinto IM, Lindholm B, et al. Epicardial fat accumulation, cardiometabolic profile and cardiovascular events in patients with stages 3-5 chronic kidney disease. J Intern Med. 2015;278(1):77-87.

13. McCullough PA, Chinnaiyan KM, Agrawal V, Danielewicz E, Abela GS. Amplification of atherosclerotic calcification and Monckeberg's sclerosis: a spectrum of the same disease process. Adv Chronic Kidney Dis. 2008;15(4):396-412.

14. O'Leary DH, Polak JF, Kronmal RA, Manolio TA, Burke GL, Wolfson SK, Jr. Carotid-artery intima and media thickness as a risk factor for myocardial infarction and stroke in older adults. Cardiovascular Health Study Collaborative Research Group. N Engl J Med. 1999;340(1):14-22.

15. Briet M, Bozec E, Laurent S, Fassot C, London GM, Jacquot C, Froissart M, et al. Arterial stiffness and enlargement in mild-to-moderate chronic kidney disease. Kidney Int. 2006;69(2):350-357.

16. Roumeliotis A, Roumeliotis S, Panagoutsos S, Theodoridis M, Argyriou C, Tavridou A, Georgiadis GS. Carotid intima-media thickness is an independent predictor of all-cause mortality and cardiovascular morbidity in patients with diabetes mellitus type 2 and chronic kidney disease. Ren Fail. 2019;41(1):131-138.

17. Zuo J, Hu Y, Chang G, Chu SL, Tan I, Butlin M, Avolio A. Relationship between arterial stiffness and chronic kidney disease in patients with primary hypertension. J Hum Hypertens. 2020;34(8):577-585.

18. Ma YC, Zuo L, Chen JH, Luo Q, Yu XQ, Li Y, Xu JS, et al. Modified glomerular filtration rate estimating equation for Chinese patients with chronic kidney disease. J Am Soc Nephrol. 2006;17(10):2937-2944.

19. Sen T, Tufekcioglu O, Ozdemir M, Tuncez A, Uygur B, Golbasi Z, Kisacik H. New echocardiographic parameter of aortic stiffness and atherosclerosis in patients with coronary artery disease: aortic propagation velocity. J Cardiol. 2013;62(4):236-240.

20. Edwards NC, Ferro CJ, Townend JN, Steeds RP. Aortic distensibility and arterial-ventricular coupling in early chronic kidney disease: a pattern resembling heart failure with preserved ejection fraction. Heart. 2008;94(8):10381043.

21. Laurent S, Boutouyrie P, Asmar R, Gautier I, Laloux B, Guize L, Ducimetiere P, et al. Aortic stiffness is an independent predictor of all-cause and cardiovascular mortality in hypertensive patients. Hypertension. 2001;37(5):1236-1241.

22. Guerin AP, Blacher J, Pannier B, Marchais SJ, Safar ME, London GM. Impact of aortic stiffness attenuation on survival of patients in end-stage renal failure. Circulation.
2001;103(7):987-992.

23. Shlipak MG, Fried LF, Cushman M, Manolio TA, Peterson D, Stehman-Breen C, Bleyer A, et al. Cardiovascular mortality risk in chronic kidney disease: comparison of traditional and novel risk factors. JAMA. 2005;293(14):1737-1745.

24. Aydin E, Altin C, Sakallioglu O, Yilmaz M, Gezmis E, Elif Sade L, Muderrisoglu H. Epicardial adipose tissue thickness and carotid intima-media thickness in hemodialysis patients. Acta Cardiol Sin. 2017;33(3):266-272.

25. Ozturk MT, Ebinc FA, Okyay GU, Kutlugun AA. Epicardial adiposity is associated with microalbuminuria in patients with essential hypertension. Acta Cardiol Sin. 2017;33(1):74-80.

26. Akbas EM, Demirtas L, Ozcicek A, Timuroglu A, Bakirci EM, Hamur H, Ozcicek F, et al. Association of epicardial adipose tissue, neutrophil-to-lymphocyte ratio and platelet-to-lymphocyte ratio with diabetic nephropathy. Int J Clin Exp Med. 2014;7(7):1794-1801.

27. Song G, Qiao W, Liu K, Yu X. Epicardial adipose tissue in patients with chronic kidney disease: a meta-analysis study and trial sequential analysis. Int Urol Nephrol. 2020;52(12):2345-2355.

28. Sheng YN, Zhao DM, Ma QL, Gao Y. [Association between epicardial fat volume and coronary artery calcification in patients with chronic kidney disease]. Zhonghua Xin Xue Guan Bing Za Zhi. 2017;45(2):121-125.

29. Ozkurt S, Karavelioglu Y, Musmul A. Echocardiographic evaluation of epicardial adipose tissue in non-diabetic, non-hypertensive hemodialysis patients. Ren Fail. 2013;35(6):891-895.

30. Turan MN, Gungor O, Asci G, Kircelli F, Acar T, Yaprak M, Ceylan N, et al. Epicardial adipose tissue volume and cardiovascular disease in hemodialysis patients. Atherosclerosis. 2013;226(1):129-133.

31. Patel ML, Sachan R, Singh GP, Chaudhary SC, Gupta KK, Atam V, Parihar A. Assessment of subclinical atherosclerosis and endothelial dysfunction in chronic kidney disease by measurement of carotid intima media thickness and flow-mediated vasodilatation in North Indian population. J Family Med Prim Care. 2019;8(4):14471452.

32. Benedetto FA, Mallamaci F, Tripepi G, Zoccali C. Prognostic value of ultrasonographic measurement of carotid intima media thickness in dialysis patients. J Am Soc Nephrol. 2001;12(11):2458-2464.

33. Turan Y, Turan E. Aortic Stiffness Index And Carotid intima-media thickness are independently associated with the presence of microalbuminuria in patients with type 2 diabetes mellitus. Diabetes Metab Syndr Obes. 2019;12:1889-1896.

34. Margekar V, Thakur S, Jatav O, Tiwari D, Gupta M, Yadav P. Carotid intimal medial thickness (CIMT) in patients of chronic kidney disease (CKD) and its association with CKD staging. Journal of Contemporary Medical Research. 2020;7(3):C7-C9. 